\title{
BTS statement on criteria for specialist referral, admission, discharge and follow-up for adults with respiratory disease
}

\author{
British Thoracic Society Standards of Care Committee
}

Correspondence to:

Dr C F A Pantin, Department of Respiratory Medicine, City General University Hospital of North Staffordshire, Newcastle Road, Stoke on Trent

Staffordshire ST4 60G, UK; charles.pantin@btinternet.com

Received 20 July 2007 Accepted 9 November 2007

\section{EXECUTIVE SUMMARY}

The UK National Health Service (NHS) is developing rapidly. During this time of change there are many resource pressures on the overall healthcare system. There are also drivers being developed which aim to improve the consistency and quality of care for all patients. The British Thoracic Society (BTS) recognises these challenges and is an advocate for high quality standards in respiratory care. This statement has been developed from major evidence-based guidelines by an expert group with wider consultation including primary care colleagues in the General Practice Airways Group. It is aimed at those who commission and quality assure respiratory care as well as those involved in delivering this care.

The BTS recognises that, in many areas, development of integrated care pathways and initiatives to improve care with earlier discharge from hospital, prevention of admission strategies and fast track outpatient clinics are developing with a variety of experiences. These are intended to improve the patient's experience of care, the quality of care and the effectiveness of the care provided.

We hope that this statement will allow positive collaboration between primary and secondary care-and discussion between clinicians and commissioners. The areas covered pertain to the commonest causes of respiratory admission in the adult population, and are relevant no matter who refers and who manages the care of the patient. We recognise that, at times, individual uncertainties in clinical practice and balanced clinical judgement will result in practice outside the guidance. However, we hope these will be relatively few and for understandable reasons.

Some areas of the statement are aimed at common problems which result in high-cost hospital admission and high health care costs; others are aimed at rarer conditions. It should be remembered that some of the rarer conditions can be a large "hidden" cost to the NHS-all of these are very important to our patients.

We hope this statement will be used by:

- service planners and developers

- commissioners of respiratory services

- providers of unscheduled care and emergency services

- clinicians in primary care (medical, nursing and others)

- clinicians in medical assessment units.

It is not aimed specifically at the following groups, but they will find it useful to read as an adjunct to the current BTS guidelines:
- clinicians in secondary care (medical, nursing and others)

- patient user groups

- audit and quality assurance departments.

The statement provides guidance on the following clinical disease areas:

- asthma

- chronic obstructive pulmonary disease (COPD)

- lung cancer and mesothelioma

- pneumonia

- pulmonary embolism

- obstructive sleep apnoea

- other rarer conditions including bronchiectasis, lung fibrosis, sarcoidosis and tuberculosis.

The guidance suggests the acceptable indications for:

- referral to a specialist respiratory clinic

- follow-up in a specialist respiratory clinic

- discharge from a specialist respiratory clinic

- emergency admission to hospital

- discharge from hospital.

The BTS recognises that practice is changing in the NHS and that this statement should be seen as providing, for the first time, good practice indicators to work towards and achieve when any respiratory service redesign is considered.

The BTS wishes to promote these as potentially auditable parameters for significant event analysis and quality assurance monitoring.

\section{INTRODUCTION}

\section{Background}

The need for this document has arisen with the changing emphasis of care in the UK NHS from hospital to community-based care. This has resulted in a drive for Primary Care Trusts (PCTs) to strengthen community care.

In the UK, Western Europe and the USA there has been emphasis on changing the way both acute and chronic diseases are managed. The degree of change differs between countries and areas within each country, but has generally shared the following aims:

- reduction in the rate of hospital attendances

- reduction in hospital admissions

- reduction in hospital length of stay

- reduction in the rate of re-hospitalisation

- strengthening care in the community

- developing care models for chronic diseases.

This document proposes to inform the debate about the management of respiratory diseases, taking account of the wide diversity of users. It is 
also intended that this guidance statement might form the basis for the creation of pathways for the management of respiratory conditions in the future.

The proposed shift of care to the community where specialist knowledge and sophisticated investigations are not routinely available has created a justifiable anxiety on the safety of patient management. Mindful of the rapid changes in healthcare provision, the BTS Standards of Care Committee has taken the view that a statement highlighting criteria for specialist outpatient referral, hospitalisation, discharge and follow-up would help the wide range of professionals involved in respiratory care to streamline the management of patients while maintaining a good and safe standard of care.

The facts in these documents are rooted in many documents established by the BTS, European Respiratory Society (ERS) and the American Thoracic Society (ATS). It is recognised that this is a rapidly developing area of care and that this guidance will need to be updated on a regular basis.

\section{Primary-secondary interface}

The NHS plan encourages the development of integrated care. The principles to allow implementation of integrated care can be summarised as follows:

- Social change and health promotion is developed to give a healthy lifestyle for the many, not the few.

- Patients (advised by the professionals) are the primary decision makers about their own health.

- Care should be given as near to the patient's home as is practical. Care needs to be rooted in primary care settings and underpinned by vastly improved communication and new partnerships across the whole health and social care spectrum.

- Care should be provided by professionals who know the patient and can form a supportive relationship. In patients with long-term conditions, this is paramount.

- Only when care cannot be provided to the appropriate clinical standards in the community should the patient be admitted to the acute hospital.

- The key to all care is the patient and professional encounter. The full health and social record must be available at this encounter for it to be effective.

This will lead to a more dispersed service.

Many patients suffer from the common respiratory long-term conditions chronic obstructive pulmonary disease (COPD) and asthma. These form a large component of those patients requiring chronic care programmes. ${ }^{1}$

The evidence that chronic care works has been reviewed. ${ }^{2}$ There is only evidence for positive outcomes in well-defined areas but overall conclusive grade 1 evidence is lacking. For example, robust studies showing that chronic management in COPD reduces the frequency of hospital admissions or unscheduled visits to the accident and emergency department need to be undertaken. ${ }^{3}$ However, pilot schemes in practices (Castlefields) or PCTs (East Lincolnshire) have given encouraging results. In East Lincolnshire the model adopted included:

- development of lead clinicians for COPD in the participating practice

- provision of a template for systematic care of patients with COPD

- adaptable education programme for participating clinicians

- regular monitoring and support practice visits by a team of primary and secondary care specialists
- establishment of COPD clinical forums for participating clinicians

- provision of equipment to participating practice (spirometer and pulse oximeter funding for specific COPD clinics in general practice).

This primary care initiative showed a $22.4 \%$ reduction (crude rate per 1000 population) for emergency COPD admissions following implementation of phases 1 and 2 (1999-2003). Figures from core practices show this to be sustained. One neighbouring PCT showed no reduction in emergency admissions and another PCT had a fall of $7.5 \%$.

In 2003 a Cochrane systematic review ${ }^{3}$ concluded that Hospital-at-Home was a safe and effective approach for selected patients with exacerbations of COPD and suggested that one in four patients presenting to hospital as an emergency would be suitable for home treatment with nursing support.

\section{The keys to success}

For this reorganisation of care to be implemented, it is vital that respiratory care is planned across a health economy, breaking down barriers between primary and secondary care. The components for success include the following.

\section{Vision}

All organisations should plan services against the principles of integrated care. Each must look at the whole health and social care economy in its entirety and what this means for the individuals in their care.

\section{Clinically-led involvement}

Patients are involved in their own care and patients and professionals set the standards for care.

\section{Care pathways, documentation and communications}

For a dispersed but integrated system of care to work, information and communication must be slick. It must cross all organisational interfaces. Indeed, for the patient the interface should be invisible-health and social care should be unified. Respiratory specialists must be involved in this work, using their expertise to aim for implementation of high standards of care across their district.

\section{METHODOLOGY}

A working party was established by the BTS Standards of Care Committee in January 2006. The steps towards formulating this document were as follows:

- Disease specific statements were produced by lead authors.

- A subcommittee of the Standards of Care Committee reviewed the initial statements.

- Each statement was sent to one of the main authors of the national guidelines of that disease-specific area.

- Each statement was refined incorporating the comments of the national leads.

- The statements were edited into one document.

- An executive summary was produced.

- The Standards of Care Committee of the BTS critically reviewed the document. The document was reviewed by council members of the BTS.

- The document was reviewed by the joint working party set up between the General Practice Airways Group (GPIAG) and the BTS aiming to "Improve and Integrate Respiratory Services in the NHS (IMPRESS)" for comment. 
- The final copy incorporated the comments of the BTS Standards of Care Committee/Council members and IMPRESS.

Whenever possible the document follows the following template. However, because some diseases are by their nature either acute or chronic, the structure of that section will reflect only the appropriate section of the template.

- The burden of disease

- ICD10 codes

- Ambulatory care

- Criteria for referral to respiratory specialists

- Criteria for follow-up in a respiratory specialist clinic

- Criteria for discharge from a respiratory specialist clinic

- Frequency of follow-up

- Acute phase of disease

- Assessment

- Hospital-at-Home

- Criteria for hospital admission

- Criteria for hospital discharge

- Criteria for follow-up in specialist care

- Criteria for discharge from follow-up in a specialist clinic

\section{ASTHMA}

\section{Burden of disease ${ }^{45}$}

The prevalence of asthma in the UK is one of the highest in Europe. Over $14 \%$ of children have asthma. Asthma poses a major problem to the patients and to the health care community.

In the UK in 2000 almost 3.9 million GP consultations for asthma took place. In 1999 there were just fewer than 74000 hospital admissions for asthma symptoms. The cost of asthma care in the UK is in the region of $£ 1.2$ billion, $£ 850$ million of which is borne by the NHS and the rest represents loss of productivity as a result of asthma.

\section{ICD10 codes}

Asthma: J45; status asthmaticus: J46.

\section{Ambulatory care}

Regular review of patients with asthma by primary care nurses, primary care physicians or secondary care physicians is associated with favourable clinical outcome including reduced school and work absence, a reduced exacerbation rate and improved symptom control. ${ }^{6-8}$

Most patients can control their asthma with regular support from primary care. All patients should be encouraged to keep a symptom and a peak flow diary.

Criteria for referral to a respiratory specialist outpatient clinic for patients with newly diagnosed asthma or pre-existing asthma ${ }^{4}$

Refer if any of the following are present:

- doubt about the diagnosis of asthma

- uncontrolled asthma symptoms despite optimal management

- suspected co-morbidity such as a component of heart failure, suspected malignancy, co-existing COPD

- difficult asthma with persistent symptoms despite the use of optimal therapy advocated at step 4 on the BTS algorithm ${ }^{4}$ and beyond

- new diagnosis in patients $>65$ years of age with multiple medical problems
- asthma requiring repeated hospital admissions due to worsening of asthma symptoms over the previous year that is deemed to need a specialist evaluation

- elevated specific plasma Ig E level raising the possibility of allergic bronchopulmonary aspergillosis

- patients receiving oral steroids or requiring treatment with immunosuppressants or monoclonal antibodies

- systemic symptoms raising the possibility of asthma being part of a vasculitic condition

- the need to confirm or rule out occupational asthma: consider occupational asthma in all adults with a new diagnosis of asthma, with the clinician alerted by a positive answer to the question: "Is your asthma better away from work?".

\section{Assessment of patients by a respiratory specialist ${ }^{4}$}

Asthma specialists aim to manage asthma and its complications using appropriate steps tailored to the patient's history and evolving investigations including:

- high-resolution CT scans if bronchiectasis or lung fibrosis is suspected or if there is an abnormality of the chest radiograph suggesting allergic bronchopulmonary aspergillosis

- oesophageal pH monitoring

- CT scan of the facial sinuses

- serial peak flow monitoring during work and for period off work for suspected occupational asthma

- refer to respiratory physiotherapists and/or a psychologist for management of hyperventilation.

- assess suitability for management with novel treatments (eg, monoclonal antibodies).

\section{Discharge from specialist clinics to primary care ${ }^{4}$}

Patients with well controlled asthma should be cared for in primary care. The following are broad criteria for discharge to primary care:

- patients who have an established diagnosis of stable asthma

- patients with well controlled hyperventilation syndrome

- occupational asthma with stable disease when patients have been successfully removed from the causative agents

- stable disease in patients who have been treated for sinusitis or gastro-oesophageal reflux.

\section{Follow-up by a specialist respiratory clinic $^{4}$}

This should be offered to selected patients. The frequency of follow-up depends on the severity of asthma and stability of symptoms and peak flow reading. Those who need a review every 2-3 months include:

- patients with repeated exacerbations

- patients with severe disease requiring multiple pharmacological agents

- patients with brittle asthma

- patients who have had a near fatal asthma attack (defined by a raised carbon dioxide tension and/or requiring mechanical ventilation with raised inflation pressures).

Those with more stable clinical disease who should be reviewed every 4-6 months by an asthma specialist include:

- patients receiving treatment with novel treatments (eg, monoclonal antibodies)

- patients with stable prednisolone-dependent asthma

- patients with stable asthma on any other immunosuppressant 
- symptomatic patients with concomitant bronchiectasis

- patients who wish to be treated by a specialist with the agreement of the GP and specialist.

\section{Discharge from ambulatory care ${ }^{4}$}

The routine clinical review of patients with asthma is associated with improved clinical outcome including reduction in the rate of asthma exacerbations. The outcome of care does not differ whether this is done by doctors, nurses or pharmacists.

It is recommended that general practitioners (GPs) and primary care nurses keep a list of patients with asthma and their medications, and that a structured clinical review takes place at least annually.

\section{Acute phase of disease}

Several confidential inquiries ${ }^{9-12}$ into deaths from asthma attacks have shown that most deaths occurred outside hospital. Most deaths were in patients with chronic asthma. Inadequate treatment and underprovision of written management plans were observed in some deaths. Lack of prompt administration of oral or inhaled corticosteroids was a major factor.

Most asthma exacerbations can be treated in the community with regular review to alert for criteria to refer to hospital.

\section{Criteria for referral to hospital during an acute exacerbation ${ }^{4}$}

- Continuation or worsening of symptoms despite treatment with inhaled and nebulised bronchodilators and oral glucocorticoids

- Signs of life-threatening asthma or acute severe asthma (see lists below).

- Lack of response to treatment, including lack of improvement of peak expiratory flow (PEF) to $>50 \%$ of best or predicted despite treatment for $24 \mathrm{~h}$.

- Severe symptoms including inability to complete sentences, impairment of consciousness or impairment of haemodynamics.

- Silent chest during auscultation raising the possibility of severe asthma or pneumothorax.

- Patients with a history of severe uncontrolled asthma who live alone or where home circumstances do not allow adequate supervision.

- Patients with an unclear diagnosis and who are acutely unwell.

\section{Life-threatening asthma: risk factors}

Patients with high-risk factors should be kept on a register known to the asthma specialist service. These include:

- previous near-fatal asthma

- asthma previously needing ventilation or associated with acidosis

- asthma controlled by more than three classes of treatment

- two or more hospitalisations over the previous year

- brittle asthma

- frequent use of $\beta_{2}$ agonists to control symptoms

- patients known to be non-compliant including clinic nonattenders, those known to have depression, those in denial or with learning difficulties

- abuse of alcohol or recreational drugs

- disadvantaged socioeconomic status.
Life-threatening asthma: clinical features

- peak expiratory flow $(\mathrm{PEF})<30 \%$ predicted

- silent chest

- cyanosis

- exhaustion

- impaired consciousness

- feeble respiratory effort

- oxygen saturation $<92 \%$.

Acute severe asthma: clinical features

- $33 \%<\mathrm{PEF}<50 \%$ of recent best or predicted

- respiratory rate $\geqslant 25 / \mathrm{min}$

- tachycardia $\geqslant 110$ beats/min

- patients unable to complete sentences in one breath.

Other reasons for hospital admission ${ }^{4}$

- abnormal chest radiograph indicating complications such as large segmental collapse due to mucus plugging, pneumothorax or pneumonia

- pregnancy

- psychological instability

- home circumstances which make care at home inappropriate.

\section{Discharge from hospital: timing and criteria $^{4}$}

- stability of symptoms on discharge medications for $24 \mathrm{~h}$

- $\mathrm{PEF}>75 \%$ of recent best or predicted and diurnal variation of $<25 \%$ unless discharge has been agreed with a respiratory physician

- patient demonstrating an understanding of their symptoms and knowing when and whom to ask for help

- patients demonstrating understanding of the purpose of their medications and competence in using inhaled medications appropriately

- patients with home circumstances that the multidisciplinary team deems to be safe and adequate to seek help upon further attacks

- appointment with GP or practice nurse within 2 weeks and/ or with a specialist respiratory physician or specialist asthma nurse within 4 weeks.

\section{Follow-up after hospital discharge ${ }^{4}$}

Routine follow-up improves outcome for patients with asthma:

- all patients must be followed up by a practice nurse or a GP

- patients' symptoms and peak flow should be assessed

- cigarette smoking status and smoking cessation counselling according to BTS guidelines for all smokers

- frequency of follow-up should be decided by the GP

- patients who needed specialist referral for uncontrolled asthma attacks should be reviewed at least once every 6 months

- all patients with asthma should be listed as high risk in each general practice.

\section{CHRONIC OBSTRUCTIVE PULMONARY DISEASE (COPD) Burden of disease ${ }^{13} 14$}

The prevalence of COPD in the UK is around $1.5 \%$. Approximately 900000 people have symptoms and signs of COPD; $7 \%$ of men over 75 years of age suffer from COPD. The 
Table 1 COPD: summary of follow-up for patients ${ }^{13}$

\begin{tabular}{|c|c|c|}
\hline & Mild to moderate COPD & Severe COPD \\
\hline Frequency of follow-up & At least annual & At least twice per year \\
\hline \multirow[t]{2}{*}{ Vaccination } & Offer annual influenza vaccination & Offer annual influenza vaccination \\
\hline & Offer pneumococcal vaccination & Offer pneumococcal vaccination \\
\hline \multirow[t]{11}{*}{ Areas assessed } & Smoking status & Smoking status \\
\hline & Management of symptoms: & Management of symptoms \\
\hline & Activity limitation due to breathlessness & Activity limitation due to breathlessness \\
\hline & Frequency of exacerbations & Frequency of exacerbations \\
\hline & Efficacy of mediations & Presence of cor pulmonale \\
\hline & Inhaler technique & Need for treatment escalation \\
\hline & Need for specialist referral & Need for long-term oxygen therapy \\
\hline & Need for pulmonary rehabilitation & Need for social services support \\
\hline & & Nutritional input \\
\hline & & Need for specialist referral \\
\hline & & Need for pulmonary rehabilitation \\
\hline \multirow[t]{4}{*}{ Measurements to make } & $\mathrm{FEV}_{1}, \mathrm{FVC}$ & $\mathrm{FEV}_{1}, \mathrm{FVC}$ \\
\hline & BMI calculation & BMI calculation \\
\hline & MRC dyspnoea score & MRC dyspnoea score \\
\hline & Oxygen saturation & Oxygen saturation \\
\hline
\end{tabular}

$\mathrm{FEV}_{1}$, forced expiratory volume in $1 \mathrm{~s}$; FVC, forced vital capacity; BMI, body mass index; MRC, Medical Research Council.

Lung Report III ${ }^{14}$ by the British Lung Foundation indicated that only one in four patients with COPD is diagnosed.

The cost of COPD is considerable. In a district serving a population of 250000 there are over 14000 consultations for COPD annually. The cost of hospitalisation for COPD in 20012 amounted to $£ 587$ million in the UK. In addition, COPD costs 20.4 million lost working days among men in the UK.

Early diagnosis, prevention and management of stable COPD should occur within primary care by trained clinicians. They should be categorised on spirometry into mild (forced expiratory volume in $1 \mathrm{~s}\left(\mathrm{FEV}_{1}\right)>80 \%$ predicted), moderate $\left(60 \%>\mathrm{FEV}_{1}>30 \%\right)$ and severe $\left(\mathrm{FEV}_{1} \leqslant 30 \%\right)$.

\section{ICD10 codes}

COPD; J44; emphysema: J43.

Table 2 COPD: criteria for referral to a specialist respiratory outpatient clinic

\begin{tabular}{|c|c|}
\hline Reason & Purpose \\
\hline $\begin{array}{l}\text { Diagnostic uncertainty and exclusion of } \\
\text { asthma }\end{array}$ & $\begin{array}{l}\text { Establish diagnosis and optimise treatment. } \\
\text { Check degree of reversibility of airflow } \\
\text { obstruction }\end{array}$ \\
\hline $\begin{array}{l}\text { Unusual symptoms such as } \\
\text { haemoptysis }\end{array}$ & $\begin{array}{l}\text { Investigate cause including exclusion of } \\
\text { malignancy }\end{array}$ \\
\hline Rapid decline in $\mathrm{FEV}_{1}$ & Optimise management \\
\hline Suspected severe COPD & Optimise management \\
\hline Onset of cor pulmonale & Confirm diagnosis and optimise treatment \\
\hline $\begin{array}{l}\text { Assessment of home oxygen therapy: } \\
\text { burst, ambulatory or long-term oxygen } \\
\text { therapy }\end{array}$ & $\begin{array}{l}\text { Optimise management, measure blood } \\
\text { gases and prescribe oxygen therapy }\end{array}$ \\
\hline $\begin{array}{l}\text { Assessing the need for pulmonary } \\
\text { rehabilitation }\end{array}$ & $\begin{array}{l}\text { Optimise treatment and refer to specialist or } \\
\text { community-based rehabilitation service }\end{array}$ \\
\hline Bullous lung disease & $\begin{array}{l}\text { Confirm diagnosis and refer to medical or } \\
\text { surgical units for bullectomy }\end{array}$ \\
\hline COPD $<40$ years of age & $\begin{array}{l}\text { Establish diagnosis and exclude } \alpha_{1} \text { - } \\
\text { antitrypsin deficiency }\end{array}$ \\
\hline $\begin{array}{l}\text { Assessment for lung transplantation or } \\
\text { lung volume reduction surgery }\end{array}$ & $\begin{array}{l}\text { Identify criteria for referral to transplant } \\
\text { centres }\end{array}$ \\
\hline Frequent chest infections & Rule out co-existing bronchiectasis \\
\hline Dysfunctional breathing & $\begin{array}{l}\text { Establish diagnosis and refer for } \\
\text { pharmacological and non-pharmacological } \\
\text { management }\end{array}$ \\
\hline
\end{tabular}

$\mathrm{FEV}_{1}$, forced expiratory volume in $1 \mathrm{~s}$; COPD, chronic obstructive pulmonary disease.

\section{Ambulatory care ${ }^{13} 15$}

Patients should be reviewed at least annually in primary care. The frequency and assessments needed during follow-up are shown in table 1.

Patients may have depression needing assessment and treatment. They should have access to self-help groups such as the Expert patient programme and Local Breathe Easy Club.

Criteria for referral to a specialist respiratory outpatient clinic ${ }^{13}$

Referral for specialist advice may occur at any stage of the disease. The criteria for referral to a specialist respiratory outpatient clinic are shown in table 2 .

Assessment of patients by a respiratory specialist ${ }^{13}$

Respiratory specialists aim to manage COPD and its complications using appropriate steps tailored to the patient's history and evolving investigations including:

- clinical history and physical examination

- chest radiograph

- detailed lung function tests including assessment of thoracic volume and gas transfer

- CT scan of the thorax if bronchiectasis, lung fibrosis, lung cancer or other alternative pathologies are suspected or if surgery is considered (eg, transplantation or lung volume reduction)

- bronchoscopy for haemoptysis or if lung cancer is radiologically suspected

- measurement of $\alpha_{1}$-antitrypsin level if a strong family history is suspected or COPD presents at a relatively young age or in the case of basal emphysema or severe bullous diseases

- echocardiogram if cor pulmonale is suspected

- assessment for provision of nebulised bronchodilators and short- or long-term oxygen therapy

- assessment of suitability for referral for lung volume reduction surgery or lung transplantation

- assessment for fitness to fly.

Discharge from respiratory specialist clinics to primary care ${ }^{13}$ This includes: 
- patients with stable symptoms in a patient in whom the diagnosis of COPD is firmly established

- patients who express a wish to be followed up in primary care after advice from the GP and specialist.

\section{Follow-up by a respiratory specialist clinic $^{13}$}

Most patients with COPD can be satisfactorily managed in primary care. Specialist follow-up should be considered for the following:

- those with frequent exacerbations

- patients on long-term oxygen therapy or nebulised bronchodilators

- patients awaiting confirmation of diagnoses other than COPD

- patients referred for lung transplantation or lung volume reduction surgery

- patients who express a wish to be followed up by respiratory specialists after advice from the GP and specialist

- patients with chronic hypoxia who are not yet on long-term oxygen therapy.

The frequency of follow-up depends on the severity and stability of symptoms. Consider discharge as soon as the patient is stable.

\section{Criteria for discharge from all ambulatory care ${ }^{13}$}

Non-smoking patients with very mild COPD with little or no exercise limitation can be discharged from follow-up but should be advised to be seen by their primary care team if the severity of their symptoms increases.

\section{Acute phase of disease ${ }^{13}$}

An exacerbation of COPD is defined as follows: "A sustained worsening of symptoms, beyond the day-to-day variation, that is acute in onset. Commonly reported symptoms are worsening of breathlessness, cough, and increased sputum production and change in sputum colour. The changes often require change in pharmacological management."

COPD exacerbations are common. They are correlated with impaired quality of life, accelerated decline in lung function and increased healthcare costs. Most deaths from COPD are attributed to COPD exacerbations.

Some COPD exacerbations can be managed at home after assessment by specialist teams, but some need additional treatment that requires hospital referral. These criteria are not absolute. Ultimately it is a matter of clinical judgement whether or not a patient should be referred to the hospital.

\section{Criteria for referral to hospital during acute exacerbations ${ }^{13}$}

- Signs of severe exacerbations: marked dyspnoea, tachypnoea, acute confusion, new onset of cyanosis, new signs of cor pulmonale

- The presence of high-risk co-morbid condition such as pneumonia, cardiac arrhythmia, congestive cardiac failure, diabetes mellitus, renal or liver failure

- Inability to eat or sleep due to symptoms

- Rapid rate of onset of exacerbation

- Patients already on long-term oxygen therapy

- Uncertainty of the diagnosis especially in the presence of other co morbidity such as heart conditions or malignancies

- The need for therapy not available at home such as oxygen therapy and nebulised agents
- Symptoms that might require intravenous treatment with antibiotics or aminophylline

- A previous or current need for non-invasive ventilation

- Inadequate home support or living alone

- Impaired physical functions or inability of patient to cope at home

- Acute or chronic impairment of cognition

- Previous exacerbations necessitating ITU admission.

Management of exacerbation at home (intermediate Hospital-atHome arrangement) ${ }^{3}$

The Hospital-at-Home arrangement is now well established practice in the UK, Western Europe, Australia and the USA. Selected patients are discharged early from hospital and are supervised by a team comprising specialist nurses and/or physiotherapists led by a respiratory specialist until they are discharged from the scheme to the care of a community matron, community $\mathrm{COPD} /$ respiratory team or their general practices.

Early discharge for patients with COPD is both cost-effective and favoured by patients. There is no evidence that it is associated with a poorer outcome than hospital management whether this is measured by change in $\mathrm{FEV}_{1}$, mortality or health-related quality of life.

Full BTS guidelines on intermediate care Hospital-at-Home in $\mathrm{COPD}^{3}$ are available. These guidelines outline suitable criteria for Hospital-at-Home care for patients with COPD as well as level of staffing, minimal competency needed for staff caring for patients at home and basic investigations required to assess patients in their home environment.

Intermediate care Hospital-at-Home during an acute exacerbation should not be offered to patients who meet the accepted criteria for hospitalisation previously listed. Patients who can be treated at home and those who should be removed from the scheme during exacerbations and referred to hospital are shown in table 3.

\section{Assessment of recovery from exacerbations for hospitalised patients $^{13}$}

Recovery from COPD exacerbations is assessed by clinical improvement combined with improvement of basic signs including respiratory rate, pulse and oxygen saturation. Arterial blood gases need to be monitored in patients admitted with exacerbations who have had hypoxia with or without hypercapnic acidosis.

Spirometry or peak flow measurement should not be relied upon as the degree of recovery is small and can lag behind clinical improvement.

\section{Recommendations at discharge $\mathrm{e}^{13}$}

- Patients should be established on discharge medications which may include short-term nebuliser therapy for at least $24 \mathrm{~h}$.

- Patients who were admitted with respiratory failure should have satisfactory oximetry or arterialised blood gases measured prior to discharge.

- All aspects of routine care need to be assessed prior to discharge.

- Patients or home carers should be given adequate information to help patients use their medications (including oxygen therapy).

- Arrangement for follow-up should be made for all patients admitted before discharge (see section on Ambulatory care). 
Table 3 COPD: criteria for suitability for Hospital-at-Home care

\begin{tabular}{ll}
\hline Patients with COPD suitable for & Patients with COPD in whom \\
Hospital-at-Home care & hospitalisation is indicated
\end{tabular}

Mild exacerbation of COPD. Increased Increase in symptoms despite treatment.

cough, sputum and mild increase in Patient unable to eat or sleep due to breathlessness. Patient is able to eat respiratory symptoms. New cyanosis or and sleep despite increase in symptoms signs of cor pulmonale developing

Mental status unchanged; patient fully Change in mental status

alert

Respiratory rate $<24 / \mathrm{min}$

Respiratory rate $\geqslant 24 / \mathrm{min}$

Patients do not have arrhythmia, clinical Signs of these are developing or othe signs of pneumonia or signs of heart systemic disease such as hypotension failure

Signs suggestive of exacerbation of COPD in patients known to have COPD

Exacerbations with preserved oxygen saturation $(>92 \%)$

Patient requiring oral antibiotics

Patient supported by family member

Mobility regarded to be adequate reduced urine output

Uncertainty of the diagnosis (eg, haemoptysis, weight loss, clinical signs of segmental collapse, pleural effusion or pneumothorax, symptoms suggestive of ischaemic heart disease or heart failure)

Exacerbations requiring oxygen therapy; oxygen saturation $\leqslant 92 \%$ in a patient not known to need long-term oxygen therapy Patients not responding to oral antibiotics who might need intravenous antibiotics

Lack of carer support (eg, hospitalisation of spouse or carers during COPD exacerbation)

Impairment or increased impairment of mobility

- Before discharge the patient, physicians and allied health professionals should be confident that patients are able to cope in their home environment otherwise extra assistance should be put in place.

- Repeat of the chest radiograph is mandatory in patients with a new chest radiographic abnormality or those who were admitted with haemoptysis to exclude thoracic malignancy.

\section{BRONCHIECTASIS (NOT DUE TO CYSTIC FIBROSIS)}

BTS guidelines on bronchiectasis are due to be published in 2008 .

\section{Burden of disease}

Bronchiectasis is defined as a dilatation and thickening of the airways manifested by cough, sputum production and frequent lower respiratory tract infection. The incidence is not known, but it has been estimated to be $1.06-1.3$ per 100000 population in the UK. It is a chronic condition so prevalence is much higher. In children, non-cystic fibrosis bronchiectasis was identified in $1 \%$ of all secondary care referrals in a UK population.

The burden of bronchiectasis has not been investigated, but general practitioners, respiratory physiotherapists and respiratory physicians recognise that patients have frequent consultations and hospitalisation during infective exacerbations, disease complications and their care at the advanced disease status.

\section{ICD10 codes}

Bronchiectasis: J47.

\section{Ambulatory care}

As in COPD, follow-up of patients with mild to moderate disease will usually take place in primary care. For patients with severe disease, local arrangements between primary and specialist care should be made.

Management to reduce infections is recommended for followup of all grades of bronchiectasis, possibly written down in an action plan:

- regular microscopy, culture and sensitivity of sputum at least yearly to inform choice of antibiotic when exacerbation occurs

- vaccinations against influenza yearly and pneumococcus 10yearly

- arrangements for appropriate antibiotics to be given early if exacerbation threatens, self-administered by the patient if necessary

- training in appropriate physiotherapy.

Criteria for referral to a respiratory specialist outpatient clinic Establishing a diagnosis: consideration should be given to evaluating a patient for bronchiectasis who presents with:

- chronic moist/productive cough, especially between viral colds or with positive bacterial cultures. Factors favouring further investigation are:

- young age at presentation

- history of symptoms over many years

- absence of smoking history

- daily expectoration of large volumes of very purulent sputum

- asthma that does not respond to treatment

- a single positive sputum culture in the setting of unexplained chronic respiratory symptoms for Staphylococcus aureus, Haemophilus influenzae, Pseudomonas aeruginosa, nontuberculous mycobacteria or Burkholderia cepacia

- an episode of severe pneumonia, particularly if there is incomplete resolution of symptoms, physical signs or radiological changes

- recurrent pneumonia

- persistent and unexplained physical signs or chest radiographic abnormalities

- local chronic obstruction of a bronchus for consideration of surgery

- respiratory symptoms in children with structural or functional disorders of the oesophagus and upper respiratory tract

- unexplained haemoptysis

- respiratory symptoms with any clinical features of cystic fibrosis (CF), primary ciliary dyskinesia (PCD) or immunodeficiency.

Follow-up by a respiratory specialist clinic

The following should be followed up at a respiratory specialist clinic:

- patients with chronic $P$ aeruginosa colonisation

- patients receiving prophylactic antibiotic therapy (oral or nebulised)

- patients with bronchiectasis and associated rheumatoid arthritis

- patients with complex deteriorating bronchiectasis with declining lung function and recurrent exacerbations

- patients on long-term oxygen therapy, nebulised bronchodilators or antibiotics

- patients referred for lung transplantation

- patients who express a strong wish to be followed up by a respiratory specialist.

Assessment of patients by a respiratory specialist

The following steps of assessment are emphasised:

- CT scan of the thorax 
- sputum for microbiology, culture and sensitivity including acid and alcohol-fast bacilli

- investigate possible causes of bronchiectasis according to age and circumstances including:

- serum immunoglobulin

- bronchoscopy for suspected malignancy and foreign bodies

- genetic profile and sweat test if cystic fibrosis is suspected

- cilia beat frequency if primary ciliary dyskinesia is suspected.

\section{Discharge from respiratory specialist clinics to primary care}

The following groups of patients can be discharged from the respiratory specialist clinic to primary care:

- patients with stable mild symptoms in whom diagnosis of bronchiectasis is established

- patients who express a wish to be followed up in the community.

\section{Criteria for discharge from ambulatory care}

Patients with very mild bronchiectasis with little or no exercise limitation or few infective exacerbations can be discharged from follow-up but should be advised to be seen by their primary care team if the severity of their symptoms increases.

\section{Acute phase of disease}

\section{Criteria for referral to hospital during acute exacerbations}

An acute exacerbation of bronchiectasis is an acute and sustained increase in symptoms that requires treatment with oral or intravenous antibiotics. Many patients with exacerbations of bronchiectasis can be managed at home, but some need additional treatment that requires hospital referral.

The criteria for hospitalisation for patients with bronchiectasis are similar to those with COPD. These include:

- signs of severe exacerbations (marked dyspnoea, tachypnoea, acute confusion, new onset of cyanosis, new signs of cor pulmonale)

- significant haemoptysis

- the need for intravenous antibiotics at a level which might require monitoring with blood tests

- inability to eat or sleep due to symptoms

- rapid rate of onset of exacerbation

- patients already on long-term oxygen therapy

- the need for treatment not available at home such as oxygen therapy and nebulised agents

- inadequate home support or living alone

- impaired physical functions or inability of patients to cope at home

- acute or chronic impairment of cognition.

Management of infective exacerbations at home (Hospital-at-Home arrangement)

Hospital-at-Home for bronchiectasis has not been evaluated to the same extent as for COPD. However, some centres offer this under the same criteria as COPD. The BTS guidelines do not recommend Hospital-at-Home for bronchiectasis.

\section{Assessment of recovery from exacerbations for hospitalised} patients

Recovery from bronchiectasis exacerbations is assessed identically to COPD. In addition, clearance of infection from the sputum is monitored by lightening of colour, decreased viscosity and amount.

\section{Recommendations at discharge}

- Patients should be established on discharge medications for at least $24 \mathrm{~h}$.

- Patients who were admitted with respiratory failure should have satisfactory oximetry or arterialised blood gases measured prior to discharge.

- All aspects of routine care need to be assessed prior to discharge. This should include an action plan of how to act in an exacerbation.

- Patients or home carers should be given adequate information to help patients use their medications (including oxygen therapy).

- Arrangement for follow-up should be made for all patients admitted before discharge (see section on Ambulatory care).

- Before discharge the patient, physicians and allied health professionals should be confident that patients are able to cope in their home environment, otherwise extra assistance should be put in place.

- A repeat chest radiograph is mandatory in patients with a new chest radiographic abnormality or those who were admitted with new unexplained haemoptysis to exclude thoracic malignancy.

\section{CYSTIC FIBROSIS}

Cystic fibrosis (CF) is recognised to be a specialist area. All patients should be managed by recognised secondary care Cystic Fibrosis Centres. Studies have found that there is a benefit in the outcome of care in favour of patients cared for by such specialist centres compared with those managed by nonspecialist units. ${ }^{16}$

\section{ICD10 code}

Cystic fibrosis: E84.

\section{COMMUNITY-ACQUIRED PNEUMONIA}

\section{Burden of disease ${ }^{17} 18$}

Pneumonia is inflammation of lung tissue which is due usually to infection with bacteria, viruses or fungi. It presents as an acute illness ranging in severity from mild to fatal. Over 66000 people died from pneumonia in the UK in 1999. In the adult population it has an annual incidence of 5-11 per 1000 population and $22-42 \%$ of these are admitted to hospital; 5$10 \%$ of adults admitted to hospital with pneumonia require ICU management.

A prevalence-based burden of illness study estimated that pneumonia incurred a direct health care cost of $£ 441$ million annually at $1992-3$ prices in the UK, and that hospital costs accounted for $87 \%$ of the total.

\section{ICD10 codes}

Influenza and pneumonia: J09-J18.

\section{Acute phase}

Decide early whether the patient can be managed at home or in hospital. For all patients, the decision to treat at home or refer to hospital is a matter of clinical judgement. 


\section{Box 1 Pneumonia: adverse prognostic factors}

Pre-existing:

- Age $\geqslant 65$

- Presence of co-existing disease

- Core (clinical adverse prognostic features) "CRB" when assessed outside hospital:

- Confusion: new mental confusion (abbreviated mental test score of $\leqslant 8$ or new disorientation in person, place or time)

- Respiratory rate $\geqslant 30$

- Blood pressure: systolic $<90 \mathrm{~mm} \mathrm{Hg}$ and/or diastolic $\leqslant 60 \mathrm{~mm} \mathrm{Hg}$

- "Additional" clinical adverse prognostic features:

- Hypoxaemia: oxygen saturation $\left(\mathrm{SaO}_{2}\right)<92 \%$ irrespective of inspired oxygen concentration

Assessment for hospital admission ${ }^{19-21}$

Referral to hospital is primarily based on clinical judgement with an assessment of severity. An aid to clinical judgement is given in box 1. The core clinical features of Confusion, Respiratory rate, Blood pressure and age are known as CRB-65.

- Patients who have a CRB-65 score of 0 are at low risk of death and do not normally require hospitalisation for clinical reasons.

- Patients who have a CRB-65 score of 1 or 2 are at increased risk of death and hospital referral and assessment should be considered, particularly with score 2 .

- Patients who have a CRB-65 score of 3 or more are at high risk of death and require urgent hospital admission.

In addition to disease severity, the three main factors influencing the decision to refer to hospital are (1) stability of pre-existing illnesses, (2) the patient's social circumstances and (3) the patient's wishes. These factors must be taken into account in all instances (box 2).

\section{Pneumonia treated in the community ${ }^{19-21}$}

Individuals with pneumonia can often be managed at home.

- Those managed at home need a review to assess disease severity (initially within $48 \mathrm{~h}$ of the primary consultation or sooner if clinically indicated) and its complications.

- Patients may require hospital admission if there is a failure to improve or they develop complications such as confusion, raised respiratory rate, hypotension, hypoxaemia or signs of complications such as septicaemia, pleural effusion/ empyema, dehydration, renal failure or lung abscess.

- In individuals whose pneumonia is treated successfully at home, specialist referral is indicated in those in whom an underlying lung disease is suspected such as chronic lung disease or lung cancer and failure of resolution of symptoms.

\section{Criteria for discharge from hospital ${ }^{19-21}$}

Those suitable for discharge should demonstrate clinical stability and usually not have more than one of the following:

- temperature $>37.8^{\circ} \mathrm{C}$

- heart rate $>100$ beats/min

- respiratory rate $>24$ breaths $/ \mathrm{min}$

- systolic blood pressure $<90 \mathrm{~mm} \mathrm{Hg}$

- oxygen saturation $<90 \%$

- inability to maintain oral intake

- altered mental status.

\section{Box 2 Pneumonia: factors influencing referral to hospital}

Development of respiratory complications: significant pleural effusion, empyema, lung abscess, metastatic infections

- Co-morbidities (eg, diabetes mellitus, chronic lung disease, malignancy, cardiac disease, renal failure, hepatic failure, cognitive impairment)

- Suspected/known carcinomatosis

- Uncertainty over diagnosis

- Vomiting

- Anorexia

- Diarrhoea

- Jaundice

- Compliance with therapy/ability to take oral medications

- Failure of antibiotic therapy

- Dehydration

- Lack of medical resources to allow review or care of patients in the community

- Social support

- Ability to perform usual activities of daily living

In reality, particularly in the elderly, many factors such as the presence of co-morbidities and social support will affect the timing of discharge from hospital. It is essential that individuals are discharged to an appropriate environment.

Criteria for follow-up after hospital admission ${ }^{19-21}$

Review is recommended around 6 weeks after hospital admission. This can be with the GP or in a specialist clinic. The hospital team must communicate with the GP regarding followup arrangements. Follow-up in a specialist clinic is usually indicated in those who have:

- persistent abnormal symptoms and physical signs

- complications of pneumonia

- high risk of malignancy (smokers)

- known or suspected underlying lung disease.

These individuals will require careful clinical and radiological assessment at follow-up. Further investigations such as bronchoscopy are recommended in this group.

\section{Discharge from follow-up ${ }^{19-21}$}

Patients can be discharged from follow-up when:

- there has been clinical and radiological resolution of the pneumonia

- resolution or treatment of complications.

\section{PNEUMOTHORAX}

This document concerns primary (spontaneous) pneumothorax (PSP) in patients with no underlying lung disease and secondary pneumothorax (SP, spontaneous pneumothorax in patients with underlying lung disease) but not pneumothorax secondary to trauma.

\section{Burden of disease ${ }^{22}$}

An average health district of 300000 can expect approximately 70 hospital admissions each year for PSP and SP combined. This is an acute condition that needs rapid assessment by the specialist respiratory team for both immediate management and also long-term advice concerning recurrence prevention. 


\section{ICD10 codes}

Pneumothorax: J93.

\section{Acute phase of disease ${ }^{22}$}

\section{Assessment}

A senior member of the respiratory team (specialist registrar or consultant) should assess all cases of pneumothorax as soon as possible. This could be in a respiratory clinic, an emergency "hot" clinic, on the medical admissions unit or in the A\&E department (this is the least preferable choice as it is associated with fewer adherences to national guidelines). The assessment should consist of:

- pulse, blood pressure and oxygen saturation measurements and chest radiography (standard posterior-anterior (PA) view is sufficient) in all patients

- arterial blood gas measurements in patients with SP

- treatment with simple aspiration or intercostal chest drain as advised by the BTS guidelines.

Patients with PSP whose symptoms resolve with simple aspiration of air from the pleural cavity may be discharged home after initial treatment with written advice to return immediately if the condition deteriorates.

\section{Criteria for hospital admission}

- All patients with a tension pneumothorax.

- Patients with PSP who fail the criteria for conservative management and/or have failed an aspiration attempt and therefore need treatment with an intercostal drain.

- All patients with SP should be admitted and observed for a minimum 24-hour period, which also allows for evaluation and treatment of the underlying lung condition.

- Wider use of thoracic vents or flutter valves may allow early discharge of patients with PSP who fulfil the criteria for hospital admission.

\section{Criteria for discharge from hospital}

- Those who have had a successful treatment with simple aspiration or intercostal drain with underwater seal (ie, resolution of symptoms and/or a rim of air $<2 \mathrm{~cm}$ ).

- For SP, the underlying condition should also have returned to a stable state (see criteria for asthma and COPD).

- All patients should be reviewed by the respiratory team, even if this is in the clinic.

\section{Criteria for surgery}

- Second or subsequent pneumothorax.

- Pneumothorax fails to resolve after 5 days in PSP or 3 days in SP, the specialist respiratory team should assess for surgery.

\section{Criteria for follow-up}

All cases should have a repeat chest radiograph and assessment in 1 month.

- This could be undertaken in primary care for PSP cases unless definitive surgery is contemplated.

- For SP, a 1-month review should be undertaken in a specialist unit to assess stability of the underlying lung disease and to discuss imaging by CT scanning with a view to thoracic surgical intervention. This will depend on the severity of the effects of the pneumothorax on the patient when admitted and their underlying conditions.
Criteria for discharge from follow-up

Primary pneumothorax

- Smoking cessation advice given

- Definitive surgery is not indicated.

\section{Secondary pneumothorax}

- The underlying lung disease is stable and definitive surgery is not indicated

- Smoking cessation advice given.

\section{LUNG CANCER AND MESOTHELIOMA \\ Burden of disease ${ }^{2324}$}

During 2001-2003 there was an average of 33346 deaths per annum from lung cancer in the UK and the annual incidence was $37514 .^{23}$ Lung cancer is the most common fatal cancer, killing 30\% more patients than breast and colorectal cancer combined. The 5-year survival is $6 \%$ for men and $7 \%$ for women. In the same period there was an average of 1720 deaths per annum from mesothelioma (more than those from cervical cancer, malignant melanoma or endometrial cancer). The incidence of mesothelioma is still rising and is expected to peak between 2010 and 2015. Between 2006 and 2020 up to 30000 people will die of the disease in the UK. ${ }^{24}$

\section{ICD10 codes}

Trachea, bronchus and lung cancer: C33-34; mesothelioma: C45.

\section{Ambulatory care ${ }^{25} 26$}

A patient who presents with symptoms, signs or investigations suggestive of lung cancer or mesothelioma should be urgently referred to a team specialising in the management of lung cancer and mesothelioma.

\section{Investigations prior to referral to specialist team}

Refer urgently for a chest radiograph a patient who presents with:

- haemoptysis

- any unexplained persistent (lasting >3 weeks) symptoms and signs shown in table 4

- unexplained changes in existing symptoms in patients with underlying chronic respiratory problems

- features suggestive of metastasis from a lung cancer (eg, in brain, bone, liver or skin)

- individuals with a history of asbestos exposure and recent onset of chest pain, shortness of breath or unexplained systemic symptoms.

The chest radiography report should be returned to the referring primary healthcare professional within 5 days of referral.

Table 4 Symptoms and signs requiring urgent referral for a chest radiograph

\begin{tabular}{|c|c|}
\hline Symptoms & Signs \\
\hline Cough & Finger clubbing \\
\hline Dyspnoea & $\begin{array}{l}\text { Cervical and/or } \\
\text { supraclavicular } \\
\text { lymphadenopathy }\end{array}$ \\
\hline Chest and/or shoulder pain & Chest signs \\
\hline \multicolumn{2}{|l|}{ Hoarseness } \\
\hline Weight loss & \\
\hline
\end{tabular}




\section{Urgent referral to specialist team}

The following groups of patients should be referred urgently to the specialist team for lung cancer and mesothelioma who must see them within 14 days of referral:

- smokers or ex-smokers aged $\geqslant 40$ years with persistent haemoptysis ( $>1$ week)

- patients with a chest radiograph suggestive of lung cancer (including pleural effusion and slowly resolving consolidation)

- patients whose chest radiograph is normal but there is a high clinical suspicion of lung cancer

- patients with stridor; if severe, they may need direct admission

- patients with signs of superior vena caval obstruction (swelling of the face and/or neck with fixed elevation of jugular venous pressure); if severe, they may need direct admission.

\section{Risk factors}

Patients in the following categories have a higher risk of developing lung cancer:

- current or ex-smokers

- smoking-related COPD

- previous exposure to asbestos

- previous history of cancer (especially head and neck).

Patients in the following categories have a higher risk of developing mesothelioma:

- worked in occupations associated with exposure to asbestos

- have a family member who may have suffered heavy exposure to asbestos.

\section{Assessment by the specialist team \\ Aims \\ - Confirm diagnosis \\ - Choose appropriate treatment \\ - Baseline values from which to monitor therapy.}

\section{Assessment}

- Full history and examination

- CT scanning

- Bronchoscopy

- Full lung function testing

- Fitness assessment

- Biopsy of tissue by radiologist and/or cardiothoracic surgeon

- Other imaging (eg, positron emission, ultrasound, bone or ventilation/perfusion scanning)

- Realistic and reliable information to enable patient to control their own life.

After assessment, all patients must be discussed at a multidisciplinary team meeting (MDT) attended by respiratory specialists, specialist nurses, oncologists, thoracic surgeons, radiologists and histopathologists. The MDT will have access to other services such as specialist palliative care.

\section{Treatment}

Treatment is broadly divided into:

- best supportive care

- active palliative treatment, eg, chemotherapy or radiotherapy

- treatment with curative intent, mainly surgery.
Follow-up

All patients require regular follow-up. Arrangements depend on the nature of the treatment and the preference of the patient and carers.

\section{Best supportive care}

Offer a specialist review clinic or ward consultation by a team specialising in lung cancer and mesothelioma which may be led by a specialist nurse. Depending on the wishes of the patient, subsequent care could be follow-up in specialist care, in the community or both. There must be a clear route back into specialist care.

\section{Active palliative treatment}

Specialist follow-up is required with appropriate specialists depending on the treatment received and the patient's needs. There should be the option of nurse-led "stock-take" clinics during this period, with the support and supervision of the MDT. Extended follow-up may be with the specialist or protocol driven nurse-led follow-up. Upon relapse or development of recurrent symptoms, the patient must be referred back to the specialist or to the lung cancer/mesothelioma MDT.

\section{Treatment with curative intent}

Specialist follow-up is required to detect complications of treatment. The duration of this will depend on the individual patient. There should be the option of nurse-led "stock-take" clinics during this period. Extended follow-up may be a combination of protocol driven nurse-led clinics, specialist follow-up and rapid assessment of new symptoms.

\section{Criteria for discharge from specialist follow-up}

Discharge should be considered in:

- Asymptomatic patients on best supportive care or completed active palliative treatment.

- Patients who have survived 5 years following curative treatment and have no symptoms or signs that suggest recurrence. Some specialists may wish to lengthen or shorten this period.

- Patients during the terminal phase of their disease who are too ill or who do not wish to attend specialist care.

Although patients with lung cancer and mesothelioma may be discharged from follow-up by the specialist team, there must be a mechanism for rapid resumption of follow-up, either by direct admission or outpatient attendance.

\section{Acute phase of disease}

Criteria for hospital admission

Patients should be admitted directly under the care of the team specialising in lung cancer and mesothelioma.

\section{Planned admission}

Patients with lung cancer and mesothelioma may require planned admission to hospital for diagnostic test(s) or treatment.

\section{Urgent review or admission}

Arrange review which may lead to admission with the specialist team at any stage in the care pathway where there is:

- uncontrolled and/or severe symptoms including pain, dyspnoea, haemoptysis, confusion, nausea and vomiting or any other symptom caused by metastatic disease or local invasion 
- signs of superior venal cava obstruction

- stridor.

\section{Criteria for discharge from hospital}

Ideally, a team specialising in lung cancer and mesothelioma will care for all inpatients. Where this is not possible, the supervising team should seek appropriate advice from the team:

- control all symptoms sufficiently to allow ongoing care in the community

- arrange appropriate community support

- give patients the opportunity to meet the lung cancer and mesothelioma nurse specialist

- give patients and relatives appropriate information about the diagnosis and intended management, including followup

- communicate clearly follow-up arrangements to the primary care team.

\section{TUBERCULOSIS \\ Burden of disease ${ }^{27} 28$}

Tuberculosis (TB) is a significant public health problem which necessitates that it is viewed in a wider context. Tuberculosis cases per 100000 reported in the UK in 2001 were: Black African 211, Pakistani 145, Indian 104, White 4.

Control and prevention of $\mathrm{TB}$ is a major priority which cannot be dissociated from treatment of the disease. The Consultant in Communicable Disease Control (CDCC) in England and Wales and the Director of Public Health/ Consultant in Public Health (CDEH) in Scotland is responsible for the control and prevention of TB.

Several key areas relating to the control and prevention of $\mathrm{TB}$ have been identified in the BTS and NICE guidelines ${ }^{27} 28$ including:

- notification to allow surveillance and initiate contact tracing of primary cases

- local organisational requirements for TB services

- measures for the control of TB in hospitals and in prisons

- protection of groups with potential exposure to TB including healthcare workers

- recognition of high rates of $\mathrm{TB}$ in the homeless

- management of TB in schools

- outbreak contingency planning

- new immigrant screening

- BCG vaccination and management of positive reactors.

Thus TB is managed by an identifiable local team experienced in TB and not in primary care. The local specialist team includes specialist physicians and nurses, microbiologists and public health professionals.

\section{ICD10 codes}

Tuberculosis: A15-19.

\section{Ambulatory care}

All individuals with suspected $\mathrm{TB}$ require urgent specialist outpatient assessment. The aims are to establish a diagnosis, institute appropriate therapy, monitor the response to therapy and assess compliance and side effects of therapy.

\section{Assessment of patients}

The assessment and diagnostic process often requires complex and invasive investigations necessitating the input of a range of specialities coordinated by a physician with experience in TB.
Although TB most commonly affects the lungs or lymph nodes, it can be disseminated throughout the body and affect several different organs such as kidney, bowel, bone and joint, central nervous system, skin, pericardium, liver. As well as simple examination of sputum or pus, specialised investigations and biopsies may be required to confirm the diagnosis and assess the extent of spread.

The purpose of the initial specialist assessment is to establish the diagnosis, assess the extent of organ involvement, severity of disease and its complications and predisposing diseases such as HIV infection.

\section{Follow-up by specialist TB team}

Subsequent treatment and continuity of care is provided by the specialist physician and specialist nurses.

Treatment is directed according to an individualised management plan. Follow-up will vary from thrice weekly directly observed therapy to a minimum monthly review for the duration of therapy. The TB specialist nurse plays an important role in education and monitoring compliance with therapy.

\section{Criteria for discharge from specialist follow-up}

Most individuals who have completed a 6-month standard treatment regimen for TB will not require follow-up. Notable exceptions to this are:

- individuals with drug-resistant TB in whom not only is the treatment course longer but follow-up is advocated for 1 year after completion of therapy to exclude recurrence of TB

- individuals who have developed complications of TB and those with HIV infection will require prolonged specialist follow-up.

\section{Acute phase of disease}

\section{Criteria for hospital admission}

Most individuals with suspected TB need urgent assessment in a specialist outpatient clinic rather than acute admission. Hospital admission for individuals with suspected or confirmed TB may be required in patients with HIV or suspected highly infectious or multidrug resistant TB. Referral for emergency hospital admission in the acute phase is primarily based on clinical judgement. TB can be disseminated or affect individual organs such as lungs, kidney, bowel, lymph node, central nervous system, skin, bone and joints, pericardium, liver. Thus, individuals can present with a variety of acute illnesses such as pneumonia, bowel obstruction, pericarditis, meningitis or seizures.

Drug regimens for $\mathrm{TB}$ involve several drugs which have potentially severe side effects such as hepatitis. The initiation of therapy can be associated with side effects that require inpatient assessment and adjustment of therapy.

\section{Criteria for discharge from hospital}

- After resolution of the acute symptoms, diagnosis and successful initiation of antituberculosis therapy.

- In those who are highly infectious, discharge from hospital is considered safe after 2 weeks of appropriate antituberculosis therapy.

- After resolution of drug-induced side effects and after established on an appropriate antituberculous regime. 
Criteria for follow-up after hospital admission

The patient continues to be cared for by the specialist TB team (see Ambulatory care).

\section{VENOUS THROMBOEMBOLIC DISEASE/PULMONARY EMBOLISM}

\section{Burden of disease ${ }^{29}$}

Pulmonary embolism (PE) has an annual incidence of 6070 cases/100 000 population, of whom 50\% develop PE as hospital inpatients or in the context of institutional care. Inhospital mortality is estimated at between $6 \%$ and $15 \%$. Proximal leg vein clot is evident in $70 \%$ of patients with pulmonary emboli without clinically apparent deep vein thrombosis (DVT).

The BTS guidelines emphasise the importance of a structured clinical assessment in directing investigation and management in order to reduce both inappropriate under-and over-diagnosis. Identifying underlying risk factors and, conversely, the presence of negative predictive features is of particular importance.

Most suspected PE occurs as an acute event, but minor or recurrent episodes may present as more insidious cardiorespiratory symptoms. The acute phase is therefore discussed before ambulatory care.

\section{ICD10 codes}

Pulmonary embolism: I26.

\section{Acute phase of disease ${ }^{29}$}

Criteria for referral to emergency medical facility

Although PE may occur as a discrete episode without significant adverse outcome, the potential for life-threatening events and the need for specialised imaging procedures dictate that, whenever acute PE is suspected, the patient is referred directly to an emergency medical facility.

\section{Presentation of pulmonary embolism}

- Sudden dyspnoea

- Haemoptysis

- Chest pain

- Sudden collapse

Pulmonary embolism: risk factor assessment Major (relative risk 5-20)

- Recent immobilisation or major surgery

- Recent lower limb trauma and/or surgery

- Clinical DVT

- Previous proven DVT or PE

- Pregnancy or postpartum

- Major medical illness

\section{Minor (relative risk 2-4) (examples)}

- Congestive cardiac failure

- Oral contraceptive, oestrogen hormone replacement therapy

- COPD

- Neurological disability

- Obesity

- Long distance sedentary travel
Criteria for discharge from emergency medical facility

In some young patients with mild symptoms and a single minor risk factor (eg, oestrogen oral contraceptive), a diagnosis of PE can be effectively eliminated by:

- dyspnoea with absence of respiratory rate $>20 / \mathrm{min}$ and without pleurisy

- normal chest radiograph

- normal oxygen tension $\left(\mathrm{PaO}_{2}\right)$, not oximetry alone.

If a definite alternative diagnosis is reached, the patient should be discharged within $4 \mathrm{~h}$ and would not require further action with regard to $\mathrm{PE}$.

However, the vast majority of patients will require additional investigation and imaging. The following tests also allow an alternative diagnosis to be made without further investigation or treatment for acute PE:

- negative validated D-dimer test

- a good quality negative CT pulmonary angiogram (CTPA)

Plus (if CTPA availability is limited):

- a good quality normal isotope lung scan (need on-site facility, normal chest radiograph and no significant concurrent cardiorespiratory disease).

\section{Criteria for management at home}

Where outpatient DVT management programmes have been established, these services provide an opportunity to offer earlier discharge from hospital or entirely outpatient treatment for patients with PE without haemodynamic effects or associated significant co-morbidity.

\section{Discharge planning and recommendations after discharge}

- Low molecular weight heparin (LMWH) unless early rapid reversal of effect might be required; given for 1 week.

- Oral anticoagulation only commenced once PE reliably confirmed.

- Target INR 2.0-3.0, then discontinue heparin.

- Standard duration of oral anticoagulation 4-6 weeks with temporary risk factor 3 months for first idiopathic PE, otherwise at least 6 months: judgement of bleeding risk versus further PE.

- Inform GP of treatment plan.

- Ensure patient is aware of side effects of anticoagulation and drug interactions.

- Provide written information on warfarin and ensure arrangements for anticoagulation supervision.

\section{Criteria for specialist follow-up}

Specialist outpatient review at 6-12 weeks and at 3 or 6 months (depending on length of warfarin therapy) is recommended for all patients in whom treatment has been initiated for PE to assess:

- response to therapy

- underlying predilection (occult cancer, thrombophilic disorder)

- proposed duration of anticoagulation therapy.

\section{Ambulatory care}

Criteria for referral

A further role for the specialist clinic relates to investigation of chronic PE, which should be considered in the differential of patients with unexplained exertional dyspnoea particularly associated with right heart failure, diffuse central chest 
discomfort or syncope. This diagnosis is easily overlooked in patients with COPD or ischaemic heart disease.

\section{INTERSTITIAL LUNG DISEASES: IDIOPATHIC PULMONARY FIBROSIS, SARCOIDOSIS AND EXTRINSIC ALLERGIC ALVEOLITIS}

\section{Burden of disease ${ }^{30}$}

More than 10000 cases of interstitial lung disease (ILD) are diagnosed in the UK each year. The most common ILDs are idiopathic pulmonary fibrosis (IPF) (sometimes called usual interstitial pneumonia (UIP) or cryptogenic fibrosing alveolitis (CFA)), sarcoidosis and extrinsic allergic alveolitis (EAA).

These three diseases dominate in terms of numbers and burden, but must be distinguished from many rarer diseases.

\section{Background to IPF}

- Prevalence 6-14.6 per 100000

- Occurs most frequently in the older population (70s), with a prevalence in those aged $>75$ years of 160 per 100000

- Prognosis worse than many cancers; more than $50 \%$ of patients will die within 3 years of diagnosis losing an average of 7 years of life (average life expectancy 3-4 years)

- No proven treatment that increases survival

- Indications for treatment: poor or declining lung function/ patient preference

- Treatment may not be appropriate in many cases owing to the presence of stable disease or co-morbidity

- Treatment should be given by a physician experienced in the use of appropriate drugs (usually a chest physician or a rheumatologist working with a chest physician).

\section{Background to sarcoidosis}

- A multisystem disease but mainly presents in the lungs

- Incidence in the UK is 2-4/100 000 new cases per year for men and 3.5-4.5/100 000 for women. Varies geographically and by age with more cases reported in Cornwall, Scotland and the North-East of England. Very few new cases over age 65 years.

- Respiratory disease divided on presentation into stage 1 (hilar lymphadenopathy alone, 91\%), stage 2 (hilar adenopathy and parenchymal involvement, $6 \%$ ) and stage 3 (parenchymal alone or fibrosis, 3\%)

- Spontaneous remission in 55-90\% for patients with stage 1 disease, $40-70 \%$ of stage 2 and $10-20 \%$ of stage 3 ; most remissions occur in the first 6 months

- As most people with pulmonary sarcoidosis will improve spontaneously, an initial period of 6 months observation is recommended

- A small number of patients get progressive disease and, in these, the mortality rate is double that for people of a similar age in the general population

- Treatment is not recommended for

- asymptomatic stage 1 disease

- asymptomatic stable mild stage 2 and 3 disease.

\section{Background to EAA}

- Examples of EAA include bird fancier's lung and occupational conditions such as farmer's lung

- Approximately 200-300 new cases of EAA each year in the UK
- If the responsible antigen (trigger) is identified and removed, the prognosis is good

- A few people develop progressive fibrosis with increased mortality

- Mainstay of treatment is removal from allergen and steroids.

\section{ICD10 codes}

Idiopathic pulmonary fibrosis: J84.1; sarcoidosis: D86; extrinsic allergic alveolitis: J67.

\section{Ambulatory care}

The majority of patients with an ILD need shared care between primary care and a specialist MDT experienced in the management of these relatively rare diseases.

The NHS Connecting for Health DOAS (Do Once and Share) Interstitial Lung Action Team suggests a specialist team of respiratory specialist doctor, specialist nurse and coordinator as developed for lung cancer care.

Criteria for initial specialist referral

All patients with ILD should be referred to a specialist clinic at the outset of their disease:

- for IPF and EAA to the respiratory clinic

- as sarcoidosis is a multisystem disease, the respiratory or specialist sarcoidosis clinic is usually the most appropriate. Depending on symptoms, the patient may be referred to other clinics such as rheumatology, dermatology, ophthalmology, cardiac or neurology.

Assessment of patients with ILD by a specialist

To obtain a diagnosis and baseline values for the commencement and future monitoring of treatment:

- chest radiography and CT scanning

- full lung function testing

- transbronchial biopsy (sarcoidosis)

- possible VATS (open) lung biopsy

- realistic and reliable information to enable patients to control their own life.

\section{Treatment}

- For EAA, remove patient from antigen (trigger)

- Discuss, withhold or commence and monitor treatment and disease progression. Treatment includes oral steroids and immunosuppressants

- If on oral steroids, bone protecting agents.

\section{Criteria for respiratory specialist outpatient follow-up} IPF

- Life-long specialist follow-up shared with GP.

\section{Sarcoidosis}

- Most patients have self-limiting disease and need follow-up for up to a year

- Patients with chronic disease usually need a life-long followup in the clinic. If the disease has been inactive for at least a year, consider discharge.

\section{$E A A$}

- The very few patients who develop progressive fibrosis 
Frequency of follow-up in outpatients for patients with ILD IPF and EAA with progressive fibrosis

- If on active therapy, monthly initially

- If stable, 3-monthly

\section{Sarcoidosis}

- Until disease stabilised, monthly initially

- If disease still active, 3-4-monthly thereafter

- If disease inactive, reduce to 6-monthly

- If disease inactive for at least a year, discharge.

Role of primary care including community care in management of ILD There are important roles for the primary care physician working together with the specialist in the management of these chronic diseases which include:-

\section{Chronic disease management}

- Support patient and carers

- Answer questions

- Communicate with specialist team

- Respite and palliative care

\section{Monitor therapy}

- If sudden decline, refer earlier

- If patient on immunosuppressants, monitor blood counts

- If on oral steroids, check on bone protecting agents

- Refer to pulmonary rehabilitation if available

\section{Acute phase of disease}

Reasons for admission to hospital

Most of the management of ILD is outpatient. Admission may be needed in the following circumstances:

- Procedures undertaken to establish the diagnosis.

- Bronchoscopy and transbronchial biopsy (day case)

- Video-assisted transthoracic lung biopsy (2-3 days stay)

- Management of sudden acute deterioration in condition

- Management of any infection/pneumothorax

- Management of chronic deterioration

- Consideration of second line therapies

- Consideration and work-up for lung transplantation

- Respite and terminal care.

\section{OBSTRUCTIVE SLEEP APNOEA SYNDROME}

\section{Burden of disease ${ }^{31}$}

The prevalence of obstructive sleep apnoea syndrome (OSAS) in middle-aged men is around $2 \%$, a similar prevalence to type 1 diabetes. The prevalence in middle-aged women is estimated at $0.5-1 \%$.

The main clinical consequence of untreated OSAS is the development of undue daytime sleepiness. This is associated with an increased risk for road traffic accidents, impairment of cognitive function and mood swings. A link with adverse cardiac and cerebrovascular events is also suspected.

The condition is now much more widely recognised, in part due to media exposure, and the association with snoring and obesity is appreciated. The great majority of patients will be seen initially in a primary care setting.

\section{Ambulatory care}

There is considerable opportunity for development of primary care-based elements of assessment and management of OSAS. Recording of sleep pattern and quality, risk factors for OSAS (persistent snoring, body mass index, hypertension, neck circumference, witnessed apnoeas and nocturnal choking episodes) along with the Epworth Sleepiness Score (ESS) are core data that could readily be acquired in primary care.

There are various models of service delivery in relation to OSAS ranging from entirely inpatient overnight diagnostic sleep study (polysomnography) and continuous positive airway pressure (CPAP) titration favoured in North America to predominantly home sleep study procedures and day visit CPAP strategies widely employed in the UK.

There is a growing acceptance that satisfactory long-term clinical outcomes can be achieved via home/outpatient OSAS services provided access to inpatient polysomnography is maintained.

Criteria for referral to a specialist sleep disordered breathing service The main purpose for specialist attendance relates to:

- evaluation of excess daytime sleepiness in relation to occupational risks (chiefly driving) and consideration of any alternative cause for daytime sleepiness

- objective confirmation of OSAS via appropriate sleep study

- advice on treatment options

- implementation and supervision of CPAP therapy

- review of CPAP poor responders or relapses.

Development of the treatment plan may include referral to:

- ENT specialist

- dental services

- weight management service

- sleep medicine service for narcolepsy.

Criteria for elective hospital admission

- Patient requires inpatient polysomnography.

Criteria for follow-up at a specialist sleep disordered breathing service

- Patients should remain under specialist review until a working diagnosis and treatment plan is established

Criteria for long term follow-up for patients with ongoing sleep disordered breathing

- CPAP initiation

- Confirmation of response to CPAP

- Maintenance of response and ensuring electrical safety of CPAP equipment

- Replacement of mask/headgear consumables.

Nurses or respiratory physiologists generally provide the CPAP service. The frequency of review and system for tracking care (eg, telephone contact, commercial servicing contract) are subject to local pressures and preference but an annual specialist attendance is recommended for those driving as an occupation

Criteria for discharge from a specialist sleep disordered breathing service

- The primary care setting could develop important supportive treatment programmes for patients with mild to moderate OSAS with nurse-led sleep hygiene education and involvement in weight management 
- In the future there is the potential for primary care participation in surveillance of patients on long-term CPAP.

\section{DEVELOPMENT GROUP MEMBERS}

\section{Document lead authors}

- Dr Nabil Jarad, Consultant Respiratory Physician, Bristol Royal Infirmary

- Dr Norman Johnson, Consultant Respiratory Physician, Whittington Hospital, Chairman of the BTS Standards of Care Committee

- Dr Charles Pantin, Consultant Respiratory Physician, Stoke on Trent

\section{Members of the Development Group Committee}

- Dr Stephen Banham, Consultant Respiratory Physician, Glasgow

- Dr Ravi Mahadeva, Consultant Senior Lecturer, Cambridge

- Dr John Harvey, Consultant Respiratory Physician, Southmead Hospital, Bristol

- Dr Steven Holmes, Chairman of the General Practice Airway Group

- Dr Martin B Allen, Consultant Respiratory Physician, Stoke on Trent

\section{Advisory group who provided opinion on specific disease areas}

- Asthma: Professor Martyn Partridge, Imperial College, London

- COPD: Professor J A Wedzicha, Royal Free Hospital, London; Dr David Halpin, Royal Devon and Exeter Hospital, Exeter; Dr Mike Rudolf, Ealing General Hospital Trust, London

- Bronchiectasis: Dr Mark Pasteur, Norwich Hospital, Norwich

- Interstitial lung disease: Dr Ian Johnston, Nottingham

- Pulmonary thromboembolic disease: Dr Tony Fennerty, Harrogate

- Tuberculosis: Professor Peter Ormerod, Blackburn

- Sleep apnoea: Professor John Stradling, Oxford

- Pneumonia: Dr Wei Shen Lim, Nottingham; Professor John Macfarlane, Nottingham

- Lung cancer: Dr David Baldwin, Nottingham

\section{Authors according to sections:}

Executive summary

- Dr Steven Holmes

- Dr Nabil Jarad

Introduction and methodology

- Dr Norman Johnson

- Dr Charles Pantin

- Dr Nabil Jarad

Primary-secondary interface

- Dr Charles Pantin

\section{Disease-specific areas}

- Asthma: Dr Nabil Jarad

- COPD: Dr Nabil Jarad

- Lung cancer and mesothelioma: Dr David Baldwin
- Pneumonia: Dr Ravi Mahadeva

- Interstitial lung diseases including sarcoidosis: Dr Norman Johnson

- Bronchiectasis and cystic fibrosis: Dr Charles Pantin, Dr Nabil Jarad

- Thromboembolic disease: Dr Steven Banham

- Sleep disorders: Dr Steven Banham

- Tuberculosis: Dr Ravi Mahadeva

- Pneumothorax: Dr John Harvey

Competing interests: None.

\section{REFERENCES}

1. Department of Health. Supporting people with long term conditions. An NHS and social care model to support local innovation and integration. London: The Stationery Office, 2005

2. Singh D. Transforming chronic care: evidence about improving care for people with longterm conditions. Health Services Management Centre, University of Birmingham, 2005.

3. BTS Standards of Care Committee. Intermediate care: Hospital-at-Home in chronic obstructive pulmonary disease. Thorax 2007:63:200-10 and www.brit-thoracic.org.uk.

4. BTS/SIGN. National guideline on management of asthma. Thorax 2003;58(Suppl 1). Updated 2004 and 2005. www.brit-thoracic.org.uk.

5. Angus R. Asthma. In: The Lung Report. Casting a shadow over the nation's health. British Lung Foundation, 2005:10-12.

6. Charlton I, Charlton G, Broomfield J, et al. Audit of the effect of a nurse run asthma clinic on workload and asthma morbidity in a general practice. Br J Gen Pract 1991:41:227-31.

7. Droogan J, Bannington K. Organisation of asthma care: what difference does it make? Nursing Times 1997:93:45-6.

8. Hoskins G, Neville RG, Smith B, et al. The link between nurse training and asthma outcomes. Br J Comm Nursing 1999;4:222-8.

9. Wareham NJ, Harrison BD, Jenkins PF, et al. A district confidential enquiry into deaths due to asthma. Thorax 1993;48:1117-20.

10. Mohan G, Harrison BD, Badminton RM, et al. A confidential enquiry into deaths caused by asthma in English health region: implication for general practice. $\mathrm{Br} \mathrm{J} G e n$ Pract 1996; 46:529-32.

11. Bucknall CE, Slack R, Godley CC, et al. Scottish Confidential Inquiry into Asthma Death (SCIAD) 1994-1996. Thorax 1999:54:978-84.

12. Burr ML, Davies BH, Hoare A, et al. A confidential inquiry into asthma death in Wales. Thorax 1999:54:985-9.

13. BTS/NICE. National clinical guideline on management of chronic obstructive pulmonary disease in adults and primary and secondary care. Thorax 2004;59/Suppl I) and www.nice.nhs.uk.

14. Calverley P. COPD. In: Lung Report III. British Lung Foundation, 1995:13-15.

15. Celli BR, MacNee B. Standard for diagnosis and treatment of patients with COPD: a summary of the ATS/ERS position paper. Eur Respir J 2004;23:932-46.

16. Kerem E, Conway S, Elborn S, Heijerman H, for the Consensus Committee. Standard of care for patients with cystic fibrosis: a European consensus. J Cystic Fibros 2005;4:7-26.

17. Macfarlane J, Lim WS. Lung infections. In: The Lung Report III. Casting a shadow over the nation's health. British Lung Foundation, 1995:22-5.

18. BTS. The burden of lung disease. London: British Thoracic Society, 2006.

19. BTS. Guidelines for the management of community acquired pneumonia in adults. Thorax 2001;56(Suppl 4):1-64 and www.brit-thoracic.org.uk.

20. BTS. Guidelines for the management of community acquired pneumonia in adults: 2004 update. www.brit-thoracic.org.uk.

21. ATS. Guidelines for the management of adults with community-acquired pneumonia Am J Respir Crit Care Med 2001:163:1730-54.

22. BTS. Guidelines for the management of spontaneous pneumothorax. Thorax 2003:58(Suppl II):iï39-52 and www.brit thoracic.org.uk.

23. Office for National Statistics. Mortality Statistics Series DH2 No 32, 2006.

24. Hodgson JT, McElvenny DM, Darnton AJ, et al. The expected burden of mesothelioma mortality in Great Britain from 2002 to 2050. Br J Cancer 2005;92:587-93.

25. NHS Executive. Referral guidelines for suspected cancer. London: Department of Health, 2000

26. National Collaborating Centre for Primary Care/NICE. Referral guidelines for suspected cancer in adults and children. NICE Clinical Guideline 27. London: NICE, 2005.

27. Joint Tuberculosis Committee of the BTS. Control and prevention of tuberculosis in the United Kingdom: code of practice 2000. Thorax 2000;55:887-901.

28. National Institute for Health and Clinical Excellence (NICE). Tuberculosis. Clinical diagnosis and management of tuberculosis, and measures for its prevention and control. NICE Clinical Guideline 33. London: NICE, 2006.

29. BTS. Guidelines for the management of suspected acute pulmonary embolism. Thorax 2003;58:470-84

30. BTS. Guidelines on the diagnosis, assessment and treatment of diffuse parenchymal lung diseases in adults. Thorax 1999;54(Suppl 1):1-28 and www.brit-thoracic.org.uk.

31. Scottish Intercollegiate Guideline Network (SIGN). Management of obstructive sleep apnoea/hypopnoea syndrome in adults. SIGN guideline 73. www.sign.ac.uk/ guidelines. 